\title{
THE RADIO AND INFRARED LUMINOSITIES OF 3CR RADIO GALAXIES -ARE THEY CORRELATED?
}

\author{
M.G. Yates \\ Department of Astronomy, University of Edinburgh, U.K. \\ L. Miller \& J.A. Peacock \\ Royal Observatory, Edinburgh, U.K.
}

\section{INTRODUCTION}

The infrared photometric study of a sample of 90 3CR radio galaxies by Lilly \& Longair (1984, hereafter LL) has demonstrated that the high redshift objects are brighter in the infrared than their low redshift counterparts; this has been interpreted as being entirely due to the evolution of their constituent stellar populations. There is however a great difference between the radio luminosities of the high and low redshift objects in this flux limited sample and we have therefore examined statistically the possibility of a correlation between the infrared and radio luminosities of these galaxies, the presence of which could bias our interpretation of the infrared Hubble diagram. We find that the radio and infrared luminosities do indeed correlate for the most powerful radio galaxies.

\section{THE ANALYSIS}

The sample of $\mathrm{LL}$ is complete in both $\mathrm{K}$ magnitude and redshift and we have defined a subset of this sample by including in our analysis only classical double FR II sources and excluding broad-lined radio galaxies. Infrared magnitudes (already corrected for galactic extinction) were taken from LL, and flux densities and spectral indices at $178 \mathrm{MHz}$ from Laing et al. (1983). The infrared magnitudes were corrected to a standard metric diameter of $43 \mathrm{kpc}$, assuming $\mathrm{H}_{0}=50 \mathrm{~km} \mathrm{~s}^{-1} \mathrm{Mpc}^{-1}$ and $\Omega_{0}=1$. Lilly \& Longair (1982) showed that the $\mathrm{K}-\mathrm{z}$ Hubble diagram is well described by the passively evolving $\mathrm{C}$ model of Bruzual (1983). This is the most natural null hypothesis to consider, although the exact amount of passive evolution is model dependent, and the observations of LL indicate that a reasonable set of parameters to choose is $\mathrm{q}_{0}=0.5$, an IMF slope $\mathrm{x}$ $=1.35$ and a formation redshift of 3.5. If the 3CR galaxies were a homogeneous set at all redshifts, subject to no other evolutionary changes the resulting corrected magnitudes would be constant for all redshifts. Figure 1 shows absolute infrared magnitude plotted against redshift for the sample. 


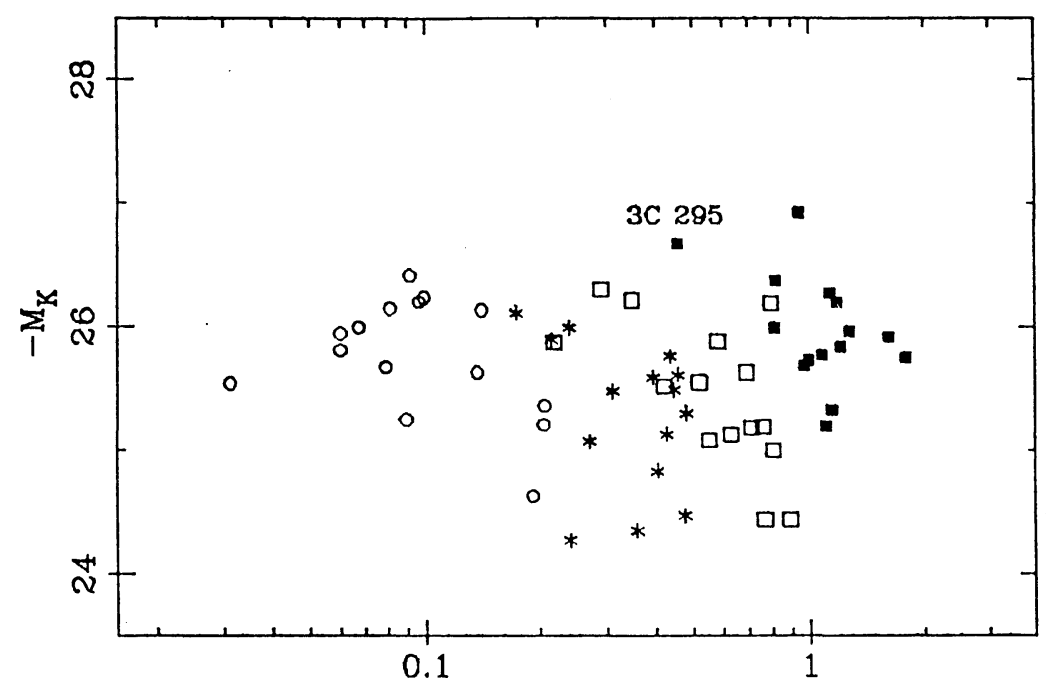

Redshift $\quad z$

Figure 1. Absolute $\mathrm{K}$ magnitude against redshift. The radio galaxies have been divided into four radio luminosity bins, each plotted with a different symbol. The filled squares are the most radio luminous galaxies, followed in decreasing radio luminosity by the open squares, the asterisks and the open circles respectively.

The relationships between absolute magnitude and radio power (M-P) and absolute magnitude and redshift $(\mathrm{M}-\mathrm{z})$ are related by the implicit relation between radio power and redshift $(\mathrm{P}-\mathrm{z})$ in a flux limited sample such as this, and so we have used a Spearmann partial rank correlation analysis to disentangle the underlying relationships, as described by Macklin (1982); this allows us to test the correlation between $M$ and $P$ in the presence of the correlation of either with $z$. The null hypothesis is that any M-P correlation is entirely due to a combination of separate $\mathrm{P}-\mathrm{z}$ and $\mathrm{M}-\mathrm{z}$ relations. If this null hypothesis can not be accepted, there must be some additional real correlation between radio luminosity and absolute magnitude, independent of redshift. A more comprehensive discussion of the partial rank analysis as applied to this data set is given in Yates et al. (1986). Table 1 presents the first and second-order (i.e. partial) coefficients for the correlated quantities, the significance calculated in terms of the Student's $t$ statistic.

Table 1 .

$\begin{array}{crrr} & \mathrm{r} & \mathrm{t} \\ \mathrm{Mz} & 0.050 & 0.379 \\ \mathrm{MP} & -0.050 & 0.379 \\ \mathrm{Pz} & 0.950 & 23.096 \\ & & & \\ \mathrm{MP}, \mathrm{z} & 0.309 & 2.456(\sim 1.5 \%) \\ \mathrm{Mz}, \mathrm{P} & -0.309 & 2.456(\sim 1.5 \%)\end{array}$


Thus the galaxies' absolute infrared magnitudes correlate with radio luminosity $(\mathrm{MP}, \mathrm{z})$ at the $1.5 \%$ significance level, and anti-correlate with redshift $(\mathrm{Mz}, \mathrm{P})$ at the $1.5 \%$ level. The similar sizes of the two correlations (although of opposite sign) are as we should expect since the magnitudes have been corrected using the $C$ model which LL empirically found to be a good fit to the data - these two correlations conspire to produce a near-zero first order correlation between $M$ and $\mathrm{z}$.

\section{DISCUSSION}

The correlation of infrared luminosity with radio luminosity has important implications for our understanding of the evolution of stellar populations in these objects since it has previously been assumed that these galaxies are a homogeneous set of standard candles. A likely mechanism for the correlation is hinted at by the bright absolute magnitude of 3C 295 ( $\mathrm{z}=0.459)$, a cD galaxy in a rich cluster and thus a galaxy which has probably undergone cannibalism (Hausman \& Ostriker 1978). At low redshifts $(z<0.2)$ classical-double radio galaxies of the sort considered here do not appear to lie in rich clusters (Longair \& Seldner 1979), however, that work did not extend to high enough redshifts to encompass the top decade of radio power which we have been able to include in our analysis here. We suggest therefore that the most powerful radio galaxies known are those rare sources which are associated with rich clusters of galaxies and which are subject to processes such as cannibalism; the most radio luminous galaxies occur at high redshift and have stellar luminosities enhanced by dynamical evolution. The first results from a deep imaging survey of the most distant $3 C R$ radio galaxies by Spinrad (these proceedings) tend to lend support to the hypothesis that the most powerful radio galaxies do indeed inhabit rich environments.

An encouraging aspect of this study concerning the use of radio galaxies to probe evolution on the Hubble diagram is that at low radio powers, classical double sources do not lie in dense environments and the effects of dynamical evolution are small. One can thus reduce the effect dynamical evolution is likely to have on the form of the Hubble diagram by only employing galaxies of moderate radio power.

\section{REFERENCES}

Bruzual, G.A., 1983. Rev. Mexicana Astron. Astrofis., 8, 63.

Hausman, M.A. \& Ostriker, J.P., 1978. Astrophys. J., 224, 320.

Laing, R.A., Riley, J.M. \& Longair, M.S., 1983. M.N.R.A.S., 204, 151.

Lilly, S.J. \& Longair, M.S., 1982. M.N.R.A.S., 199, 1053.

Lilly, S.J. \& Longair, M.S., 1984. M.N.R.A.S., $211,833$.

Longair, M.S. \& Seldner, M., 1979. M.N.R.A.S., 189, 433.

Macklin, J.T., 1982. M.N.R.A.S., 199, 1119.

Yates, M.G., Miller, L. \& Peacock, J.A., 1986. M.N.R.A.S., 221, 311. 


\section{DISCUSSION}

WAMPLFR: One can create a correlation between the absolute radio luminosity and the absolute optical luminosity by using a wrong value for $q_{0}$. Since your correlation is rather weak could a big change in $\mathrm{q}_{\mathrm{o}}$ remove it?

YATES: The partial rank analysis we have employed allows us to examine the correlation of absolute magnitude with radio luminosity independent of redshift - any magnitude redshift correlation is removed and thus a big change in $\mathrm{q}_{0}$ will not significantly change the strength of the effect seen here. 